\title{
Assessment of Cognitive Function Before and After Surgery for Posterior Cranial Fossa Lesions Using Computerized and Conventional Tests
}

\author{
Shinya ICHIMURA*, Takayuki OHIRA*, Masahito KOBAYASHI*,**, \\ Tadashige KANO*, Takekazu AKIYAMA*, Maaya ORII*, \\ Atsushi FUKUNAGA*, and Takeshi KAWASE* \\ * Department of Neurosurgery, Keio University School of Medicine, Tokyo; \\ **Department of Neurosurgery, Saitama Medical University, Saitama
}

\begin{abstract}
Cognitive function has not been well studied after neurosurgery for posterior fossa lesions despite its potential importance in determining surgical indications and approaches. The present study evaluated changes in cognitive functions after posterior fossa surgery to detect any differences between the middle cranial fossa and lateral suboccipital approaches in $\mathbf{5 0}$ patients with posterior fossa lesions such as tumors and vascular diseases. Twenty-five patients underwent surgery via the middle fossa and 25 via the lateral suboccipital approaches. Computerized test battery (CogState ${ }^{\mathrm{TM}}$ ) and conventional neuropsychological tests (serial seven-word learning test and mini-mental state examination) were examined before, 1 month after, and 3 months after surgery. All scores of the neuropsychological tests remained within normal limits after surgery. However, the scores of one computerized test battery and serial sevenword learning tests decreased significantly 1 month after surgery and recovered within 3 months, indicating temporary deterioration of short-term memory in the middle fossa group. The computerized tests detected significantly larger numbers of patients with worsened results than the conventional tests. The middle fossa approach and operation time showed correlations with the postoperative neuropsychological declines. The computerized tests could be performed easily and were beneficial for detecting subtle changes of the cognitive function after surgery. Cognitive function, especially short-term memory, may decline temporarily with the middle fossa approach and long operation time.
\end{abstract}

Key words: neuropsychological evaluation, higher brain function, computerized test, before and after surgery, skull base surgery

\section{Introduction}

Detection of asymptomatic intracranial lesions, which may require some form of surgical intervention, has recently increased because of the introduction of improved neuroimaging methods. The development of skull base surgery in the last two decades now allows good surgical results for skull base lesions. ${ }^{21,22,39)}$ Recent clinical, experimental, and neuroimaging studies have indicated that the cerebellum is involved in various neural processes of the higher brain functions such as language, working memory, executive function, and emotion. ${ }^{2-4,7,12,17,18,25,33,34,37,40)}$ Therefore, both neurological status and higher brain function must be maintained after skull base surgery. Cognitive impairment is a common consequence of infratentorial stroke, ${ }^{18,25)}$ and the neuropsychological outcomes after neurosurgical procedures for unruptured aneurysms have been investigated. ${ }^{15,16,23,29)}$ These studies have applied conventional tests, such as the mini-mental state examination (MMSE) and the Wechsler Adult Intelligence Scale-Revised (WAISR). Recently, computerized tests have been established as a tool to measure cognitive function, and can be applied in the clinical setting because of the convenience, sensitivity, and usefulness. ${ }^{5,9,10,13,35,36)}$

Posterior fossa lesions are often life threatening but are also occasionally identified as asymptomatic or with only subtle symptoms. The middle cranial

Received August 21, 2009; Accepted November 5, 2009

Author's present address: S. Ichimura, M.D., Department of Neurosurgery, Saiseikai Yokohamashi Tobu Hospital, Yokohama, Kanagawa, Japan. 
fossa and the lateral suboccipital approaches are typical neurosurgical corridors for the treatment of posterior fossa lesions. However, both approaches involve some intraoperative compression of the temporal lobe or cerebellum. The present study examines the changes in higher brain function after neurosurgical procedures for posterior fossa lesions, using both conventional and computerized tests.

\section{Clinical Materials and Methods}

The study included 50 patients, 23 females and 27 males aged 23 to 72 years (mean 50.7 years), with posterior cranial fossa lesions, including tumors without brain invasion, unruptured aneurysms, hemifacial spasm, and trigeminal neuralgia (Table 1). All patients underwent initial surgery in our institute. All patients were right handed and had no cognitive dysfunction before surgery. The middle fossa approach was applied in 25 patients and the lateral suboccipital approach in the other 25 patients. The mean lesion sizes in the middle fossa and lateral suboccipital groups were 28.6 and 24.4 $\mathrm{mm}$, respectively. No significant differences were found between the two groups regarding age and sex distribution, or the lesion side and size. In the statistical analysis, the lesion sizes of trigeminal neuralgia and hemifacial spasm were considered to be zero. All patients gave informed consent to participate in this study, which was approved by our local Institutional Review Board.

The surgical indication and approach for each patient was determined in preoperative conferences with careful evaluation of the general condition and age, size, and location of the lesion, and the operation time. The mean operation time in the middle fossa group (328.6 min) was significantly longer than that in the lateral suboccipital group (163.8 $\min )(p<0.01)$. Neuropsychological examinations were performed for all patients by one of three ex-

Table 1 Demographic and clinical data of the study subjects

\begin{tabular}{lcc}
\hline & $\begin{array}{c}\text { Middle fossa } \\
\text { approach group }\end{array}$ & $\begin{array}{c}\text { Lateral suboccipital } \\
\text { approach group }\end{array}$ \\
\hline Mean age (range), yrs & $47.5(23-71)$ & $53.8(27-72)$ \\
Sex (male/female) & $14 / 11$ & $13 / 12$ \\
Side (right/left) & $13 / 12$ & $11 / 14$ \\
Pathology & & \\
brain tumor & & 5 \\
$\quad$ meningioma & 9 & 12 \\
$\quad$ schwannoma $\quad$ chordoma & 13 & 0 \\
$\quad$ unruptured aneurysm & 1 & 0 \\
trigeminal neuralgia & 0 & 1 \\
hemifacial spasm & 0 & 7 \\
\hline
\end{tabular}

perienced operators, who were unaware of the clinical data. The examinations consisted of one computerized and two conventional tests, and all patients were assessed three times, before, 1 month after, and 3 months after surgery. The time taken to complete the examinations for each patient was about 30 minutes.

\section{Computerized test battery}

The computerized test battery, a subset of tests from CogState ${ }^{\mathrm{TM}}$ (CogState Ltd., Melbourne, Australia), was selected because of its brevity and utility for within-subjects experimental designs and for the parametric properties of the outcome measures. ${ }^{36)}$ The tests described below have previously been used in numerous psychopharmacological trials and detailed descriptions can be found elsewhere. ${ }^{6,8,26)}$ The tasks of this test battery have the form of card games and were presented on one desktop computer (Dell Inspiron 9400; Dell Inc., Round Rock, Tex., U.S.A.). At the beginning of the tasks, written instructions appeared on the left of the screen to indicate the task rules. Each subject was then given an interactive demonstration and, once they had successfully completed an adequate number of practice trials to demonstrate their awareness of the rules, the task began. The performance measurements recorded in each trial for each task were the speed of response (i.e., reaction time recorded in milliseconds) and the accuracy of response (i.e., correct or incorrect). ${ }^{36)}$ The four tasks employed in this study are described below.

Learning one-card task: In this task of learning and memory, a single 'playing card' was presented in the middle of the computer screen. The subjects were instructed to press the 'YES' key if they had seen the card before, or to press the 'NO' key if they had not. Subjects were required to learn that a series of six cards would be repeated pseudo-randomly throughout the task, intermixed with 8 distractor (i.e., non-repeating) cards in epochs of 14 cards. Three 14-card epochs were presented, and this task continued until the subject had made 42 complete responses or the maximum time allowed had elapsed (3 min). This task estimates mainly visual learning and short-term memory. ${ }^{6,36)}$

Simple reaction task: A card was presented facedown in the center of the computer screen. When this card turned face-up, participants were required to press the 'YES' key as quickly as possible. This task continued until 25 correct responses or the maximum time allowed had elapsed ( $2 \mathrm{~min}$ ). This task has estimates processing. ${ }^{10,13,36)}$

Choice reaction task: This task was similar to the simple reaction task. The subjects were asked to 
press the 'YES' key as quickly as possible whenever a red card appeared on the computer screen, and to press the 'NO' key whenever a black card appeared. This task continued until 25 correct responses or the maximum time allowed had elapsed $(2 \mathrm{~min})$. This task estimates visual identification. , $^{6,10,13,36)}$

Continuous monitoring task: Five face-up cards were presented in a row across the center of the screen. Two horizontal white lines were also presented on the screen. The five cards moved randomly in either an upwards or downwards direction. The directions of each card were independent of the others. Participants were required to monitor the movement of the cards and press the 'YES' key when any one of the cards touched one of the horizontal lines. Responses that were made before cards touched the line were classified as errors. This task continued until 25 correct responses or the maximum time allowed had elapsed ( $2 \mathrm{~min}$ ). This task estimates continuous divided attention. ${ }^{13,36)}$

\section{Conventional test battery}

The following two conventional tests were selected because they were commonly used in previous reports $^{14-16,19,20,29)}$ and recommended in the consensus statement. ${ }^{35}$

Serial seven-word learning test: This test is a recent memory test of verbal materials and consists of recalling seven unrelated words (such as ship, mountain, dog, river, forest, night, bicycle). An examiner speaks these seven words loudly and clearly to a subject, who is instructed to repeat these seven words immediately. The examiner repeats these seven words until the subject can recall all the seven words correctly. The number of times the examiner has to repeat the words represents the score of this examination. A score of one and two is considered to be normal in healthy individuals. ${ }^{19,20)}$

MMSE: This test consists of five subtests including orientation, registration, calculation, recall, and language, with a maximum score of 30 . The MMSE is suitable for screening posterior cerebral function. ${ }^{14-16,29)}$ A score of less than 24 is an indication of dementia. ${ }^{14-16,29)}$

\section{Cerebral blood flow (CBF) study}

Nine patients from each of the middle fossa and lateral suboccipital groups participated in a CBF study using single photon emission computed tomography (SPECT). The tracer, 99m-technetiumethyl cysteinate dimer (925 MBq), was intravenously administered into the patient 15 minutes after intravenous injection of acetazolamide $15 \mathrm{mg} / \mathrm{kg}$. Projection data were acquired with a continuous rotation scan of 15 minutes and reconstructed into a
$128 \times 128$ matrix with filtered back projection. The SPECT studies were performed before and 1 month after surgery. The difference between two studies was judged to be 'unchanged' or 'decreased' by a neuroradiologist unaware of the clinical data.

\section{Statistical analysis}

An analysis of variance (ANOVA) test with repeated measures was applied to assess the serial changes of the results of the neuropsychological examinations in each group. If the repeated measured ANOVA demonstrated significant effect of the time course or significant interaction between the patient groups, the Bonferroni/Dunn procedure was applied as a post hoc test. Chi-squared tests were performed to compare detection rates of decreases in the computerized and conventional tests. Stepwise regression analysis was performed to detect factors that might affect postoperative cognitive functions, such as sex, side of lesions, operation time, lesion size, and surgical approaches. The differences between the preoperative and 1 month postoperative scores were used for this regression analysis. The unpaired $t$-test was performed to assess any difference in the changes of the neuropsychological examinations between the patients with 'unchanged' and 'decreased' $\mathrm{CBF}$ rates. Statistical analyses were performed using StatView version 5.0 (SAS Institute Inc., Cary, N.C., U.S.A.) on a personal computer. Differences were considered significant if $\mathrm{p}<0.05$.

\section{Results}

No patient developed neurological deficits after surgery, and all could undergo the neuropsychological examinations as planned. Postoperative $\mathrm{T}_{2}$-weighted magnetic resonance imaging detected no brain damage such as edema, contusion, or atrophy in any patient.

Although the patients showed some changes in their postsurgical neuropsychological examination scores, all scores remained within normal limits and did not show any prominent deterioration. The detailed results of each neuropsychological examination were as follows.

\section{Computerized test battery}

Learning one-card task: ANOVA with repeated measurements revealed an effect on the reaction time $(F(2,96)=10.127, p<0.01)$ and an interaction between the time course and approach $(F(2,96)=$ 6.932, p<0.01) (Fig. 1). The post hoc tests showed that the reaction time was longer 1 month after surgery compared with before and 3 months after surgery in the middle fossa group $(\mathrm{p}<0.01)$, but no sig- 
nificant prolongation in the lateral suboccipital group. Therefore, the reaction time was prolonged temporarily but significantly after surgery, and recovered within 3 months only in the middle fossa group.

ANOVA with repeated measurements showed an effect on the accuracy of the reaction $(F(2,96)=5.51$, $\mathrm{p}<0.01$ ) and an interaction between the time course and approach $(F(2,96)=3.23, p<0.05)$ (Fig. 2). The post hoc tests demonstrated that the accuracy of reaction declined significantly 1 month after surgery compared with before and 3 months after surgery in the middle fossa group. On the other hand, there was no significant degradation after surgery in the lateral suboccipital group. Therefore, the accuracy of reaction deteriorated temporarily but significantly

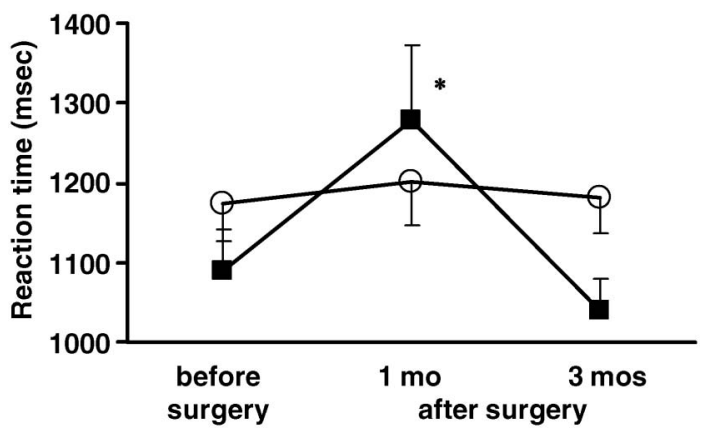

Fig. 1 Changes in the reaction times in the computerized tests performed before and after surgery. In the middle fossa group (squares), the reaction time significantly increased 1 month after the surgery, but recovered 3 months after the surgery $\left({ }^{*} p<0.01\right)$. There was no significant post-surgery prolongation in the lateral suboccipital group (circles). The vertical bars indicate standard errors.

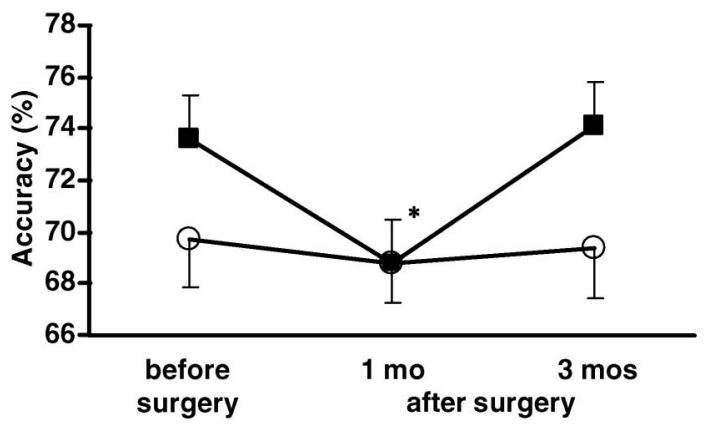

Fig. 2 Changes in the accuracy scores of the computerized tests performed before and after surgery. In the middle fossa group (squares), the reaction accuracy significantly degraded 1 month after the surgery, but recovered 3 months after the surgery $\left({ }^{*} p<0.01\right)$. There was no significant degradation in the lateral suboccipital group (circles). The vertical bars indicate standard errors. after surgery, and recovered within 3 months after surgery only in the middle fossa group.

Simple reaction, choice reaction, and continuous monitoring tasks: These tests detected no significant changes in the reaction time and accuracy.

\section{Conventional tests}

Serial seven-word learning test: ANOVA with repeated measurements revealed an effect on the score of the serial seven-word learning test $(F(2.96)$ $=3.611, \mathrm{p}<0.05)$ and an interaction between the time course and approach $(\mathrm{F}(2.96)=3.185, \mathrm{p}<0.05)$ (Fig. 3). The post hoc tests showed that the scores of the serial seven-word learning test significantly worsened 1 month after surgery compared with before and 3 months after surgery in the middle fossa group. On the other hand, there was no significant degradation after surgery in the lateral suboccipital group. Therefore, the scores of the serial seven-word learning test degraded temporarily but significantly, and recovered within 3 months after surgery only in the middle fossa group.

MMSE: ANOVA with repeated measurements showed a significant effect of the time course on the score of MMSE $(F(2,96)=5.30, p<0.01)$, but no significant difference and interaction between the two groups $(F(2,96)=1.03, p>0.05)$ (Fig. 4). The post hoc tests detected a significant increase of the scores 3 months after surgery compared with before surgery. Therefore, both groups showed better MMSE scores indicating a practice effect at 3 months after surgery.

\section{Sub-analyses}

The middle fossa group included a larger number of the patients with tumors than the lateral suboccip-

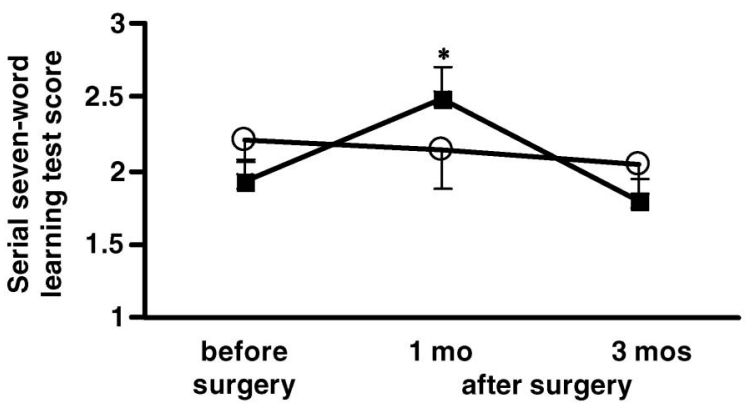

Fig. 3 Changes in the scores of the serial seven-word learning test before and after surgery. In the middle fossa group (squares), the score of the seven-word learning tests significantly degraded 1 month after the surgery, but recovered 3 months after the surgery $\left({ }^{*} p<0.01\right)$. There was no significant post-surgery degradation in the lateral suboccipital group (circles). The vertical bars indicate standard errors. 


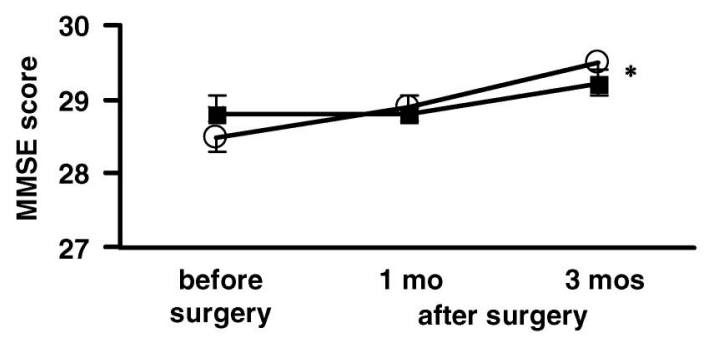

Fig. 4 Changes in the mini-mental state examination (MMSE) scores before and after surgery. In both the middle fossa (squares) and lateral suboccipital groups (circles), the MMSE scores 3 months after surgery had significantly improved in comparison with the scores before surgery $\left({ }^{*} p<0.01\right)$. The vertical bars indicate standard errors.

ital group. To minimize the effect of the tumor compression on cognitive function, sub-analyses were performed only for the patients with the brain tumors (middle fossa 24 cases, lateral suboccipital 17 cases), and obtained similar results. Both reaction time and the accuracy of reaction of the computerized test declined significantly 1 month after surgery in the middle fossa group, but not in the lateral suboccipital group. The scores of the serial sevenword learning test significantly worsened 1 month after surgery in the middle fossa group, but not in the lateral suboccipital group. The MMSE scores were significantly higher 3 months after surgery than before surgery in both groups. The data of the patients with the tumors before, 1 month after, and 3 months after surgery were as follows. In the computerized test battery (learning one-card task), the mean values of the reaction times were 1085.1, 1276.4 , and $1034.8 \mathrm{msec}$ in the middle fossa group, and 1213.0, 1233.4, and $1214.1 \mathrm{msec}$ in the lateral suboccipital group. The mean accuracies of reaction were $73.8 \%, 68.9 \%$, and $73.9 \%$ in the middle fossa group, and $71.2 \%, 70.8 \%$, and $70.9 \%$ in the lateral suboccipital group. As for the conventional tests, the mean values of the serial seven-word learning test are 1.92, 2.42, and 1.75 in the middle fossa group, and $2.35,2.29$, and 1.71 in the lateral suboccipital group. The mean scores of the MMSE are 28.9, 28.8, and 29.3 in the middle fossa group, and 28.7, 28.9, and 29.8 in the lateral suboccipital group.

\section{Comparison of computerized and conven- tional tests}

More than $70 \%$ of the patients showed prolonged response time and decreased accuracy in the computerized tests, whereas 19 (seven-word test) or 12 (MMSE) of the 50 patients had worsened scores in the conventional tests (Table 2). The computerized tests detected significantly more patients with
Table 2 Results of the computerized and conventional tests

\begin{tabular}{lllll}
\hline & \multicolumn{2}{c}{ Computerized tests } & & \multicolumn{2}{c}{ Conventional tests } \\
\cline { 2 - 3 } & $\begin{array}{c}\text { Response } \\
\text { time }\end{array}$ & Accuracy & $\begin{array}{c}\text { Serial } \\
\begin{array}{c}\text { Seven-word } \\
\text { learning } \\
\text { test }\end{array}\end{array}$ & MMSE \\
\hline Unchanged/improved & 15 & 13 & 31 & 38 \\
Decreased/worsened & $35^{*}$ & $37^{*}$ & 19 & 12 \\
\hline
\end{tabular}

*In comparison with the conventional tests, the computerized tests detected significantly higher numbers of decreased or worsened scores $(p<0.05)$. MMSE: minimental state examination.

decreased or worsened scores than the conventional tests $(\mathrm{p}<0.05)$. All patients with worsened scores in the conventional tests showed either or both prolonged response time and decreased accuracy in the computerized tests. Therefore, the computerized tests detected all patients who showed worsened scores in the conventional tests. The conventional tests detected no items which correlated with the worsened scores.

\section{Prognostic factors in the postoperative neu- ropsychological status}

The reaction time and accuracy in the computerized test batteries and the score of the serial sevenword learning test declined after the middle fossa approach. Multivariate analysis showed that the middle fossa approach was statistically associated with worsened (prolonged) reaction time. In addition, the operation time was also associated with deterioration of the accuracy of the one-card learning test. There were no other prognostic factors correlated with the postoperative neuropsychological function, including sex, age, lesion side, and mean lesion size.

\section{Change in CBF after surgery}

Five of the 9 patients in the middle fossa group showed decreased CBF in the temporal lobe of the approach side. Four of the 9 patients in the lateral suboccipital group showed decreased CBF in the cerebellar hemisphere of the approach side, but not in the cerebrum. Both computerized test batteries and conventional tests detected no significant differences in the postoperative results between the patients with normal and decreased CBF rates.

\section{Discussion}

The present study clearly showed that cognitive function, especially short-term memory, may be affected after surgery for the posterior fossa lesions, 
but such changes will be subtle and will recover within 3 months. Sensory information first passes through the appropriate sensory register before recording in a verbal format and temporarily residing in short-term storage. ${ }^{1)}$ The Papez circuit is generally recognized to be important in memory. ${ }^{28,30)}$ A recent functional imaging study also supports the view that relational memory depends upon distinct encoding operations within the medial temporal lobe, especially the hippocampus. ${ }^{11)}$ Tumor compression of the Papez circuit would cause shortterm memory disturbance. ${ }^{28)}$ In the middle fossa or lateral suboccipital approach, retraction of the medial temporal lobe or cerebellum is essential to approach the target lesion. In addition to the medial temporal lobe, the cerebellum is also involved in memory processing. ${ }^{2-4,7,33,37,40)}$ Delayed recall of verbal and visual information will be frequently impaired after subtentorial strokes. ${ }^{18)}$ However, the present study showed that retraction of the temporal lobe caused a decline in temporary short-term memory but the lateral suboccipital approach did not. The deterioration was subtle and transient, and the status of all affected patients recovered to preoperative levels within 3 months after surgery. These changes were only detectable by the computerized and serial seven-word learning tests.

The present study also showed that the operation time was correlated with the temporary deterioration of the accuracy of the learning one-card task. The operation time in the middle fossa group was significantly longer than that in the lateral suboccipital group, because of the resection of the petrous temporal bone. The longer operation time for the middle fossa approach is an important consideration, because of the possible cognitive decline, and also because an extended operation time in the lateral suboccipital approach may also cause deterioration of the cognitive function. Brain retraction may be more critical for the brain function rather than overall operation time. The present study of a limited number of the patients found brain retraction was difficult to estimate precisely due to various parameters including time, extent, direction, and intermission of retraction. However, neurosurgeons must consider the possibility of memory impairment, even slight and transient, induced by surgery for posterior lesions.

The present study examined CBF in only a limited number of the patients. Statistically, the changes in the CBF did not show any significant correlation with the higher brain function, but more than half of the patients showed decreased CBF after surgery. Temporary neuropsychological deterioration and $\mathrm{CBF}$ reduction were reported after asymptomatic aneurysm surgery. ${ }^{15)}$ Such decrease in CBF could reflect the effect of brain retraction during surgery and cause temporary cognitive dysfunction.

The conventional view is that the core functions of the cerebellum consist of regulation of motor coordination, balance, and motor speech. However, neuroanatomical, neuroimaging, and clinical studies have successfully demonstrated the functional role of the cerebellum in cognitive and affective regulation including memory. ${ }^{2-4,7,12,17,33,34,37,40)}$ The present study observed no remarkable postoperative decline in short-tem memory after the lateral suboccipital approach. However, long operation time was found to be an independent factor associated with the postoperative decline of short-term memory, suggesting that short-term memory processing can be affected by long-lasting retraction of the cerebellum.

The phenomenon of cognitive decline after surgery, i.e. postoperative cognitive dysfunction, is observed after anesthesia for weeks or months after surgery. ${ }^{24,31)}$ This phenomenon includes deterioration of intellectual function manifesting as impaired memory or concentration in variable surgery ${ }^{27,32)}$ and is often seen among the elderly population. ${ }^{31)}$ However, the present study found no correlation between cognitive impairment and age, presumably because of the low number of patients, especially the elderly, who are not usually candidates for skull base neurosurgery.

Conventionally, paper-and-pencil neuropsychological tests have been used for pre- and postoperative evaluation of higher brain functions. ${ }^{15,16,23,29)}$ The present study also applied computerized tests in addition to the conventional tests. We found that both tests were useful, but the computerized tests may have some advantages since the response time and accuracy can be measured and quantified precisely. The scores of the conventional tests are represented by integers with relatively narrow ranges and precise measurement of the reaction time is impossible. Therefore, the computerized tests detected significantly more patients with some decline after the surgery than the conventional tests. The computerized tests might also be useful for evaluation of the patients after surgery because of the quick and effortless completion. The WAIS-R and Wechsler Memory Scale-Revised, for example, take approximately 90 and 60 minutes, respectively, to complete and could impose considerable burdens on patients after surgery, whereas the computerized test takes only 15-20 minutes. ${ }^{10)}$

The present study observed practice effects in the MMSE scores. Repeated assessments with the same conventional test often lead to an improvement in 
the performance, which can obscure the precise changes in the central nervous system function. ${ }^{6,8,38}$ The computerized test, CogState ${ }^{\mathrm{TM}}$, used in this study has been developed specifically for situations that require repeated tests to provide valid measurements of a wide range of cognitive functions. ${ }^{10)}$ This test battery uses novel visual and verbal stimuli to ensure that the assessment is culture-neutral and not limited by the subject's level of education or socioeconomic background, and so can be adopted to minimize the practice effect. ${ }^{10,13)}$

In summary, surgery for posterior fossa lesions can be safely performed although temporary, slight neuropsychological deterioration may be observed after the middle fossa approach. Any such possible postoperative cognitive decline can be expected to recover within 3 months. The computerized test battery provides an easily accessible and precise method to detect changes in the neuropsychological status.

\section{References}

1) Atkinson RC, Shiffrin RM: The control of short-term memory. Sci Am 225: 82-90, 1971

2) Baillieux H, De Smet HJ, Paquier PF, De Deyn PP, Marien P: Cerebellar neurocognition: insights into the bottom of the brain. Clin Neurol Neurosurg 110: 763-773, 2008

3) Baillieux H, Verslegers W, Paquier P, De Deyn PP, Marien P: Cerebellar cognitive affective syndrome associated with topiramate. Clin Neurol Neurosurg 110: 496-499, 2008

4) Ben-Yehudah G, Guediche S, Fiez JA: Cerebellar contributions to verbal working memory: beyond cognitive theory. Cerebellum 6: 193-201, 2007

5) Cairney S, Maruff P, Clough AR, Collie A, Currie J, Currie BJ: Saccade and cognitive impairment associated with kava intoxication. Hum Psychopharmacol 18: 525-533, 2003

6) Catron DW: Immediate test-retest changes in WAIS scores among college males. Psychol Rep 43: 279-290, 1978

7) Chein JM, Fissell K, Jacobs S, Fiez JA: Functional heterogeneity within Broca's area during verbal working memory. Physiol Behav 77: 635-639, 2002

8) Chelune GJ, Naugle RI, Lüders H, Sedlak J, Awad IA: Individual change after epilepsy surgery: Practice effects and base-rate information. Neuropsychology 1 : 41-52, 1993

9) Collie A, Darekar A, Weissgerber G, Toh MK, Snyder PJ, Maruff P, Huggins JP: Cognitive testing in earlyphase clinical trials: development of a rapid computerized test battery and application in a simulated Phase I study. Contemp Clin Trials 28: 391-400, 2007

10) Collie A, Maruff P, Darby DG, McStephen M: The effects of practice on the cognitive test performance of neurologically normal individuals assessed at brief test-retest intervals. J Int Neuropsychol Soc 9: 419428, 2003

11) Davachi L: Item, context and relational episodic encoding in humans. Curr Opin Neurobiol 16: 693-700, 2006

12) de Ribaupierre S, Ryser C, Villemure JG, Clarke S: Cerebellar lesions: is there a lateralisation effect on memory deficits? Acta Neurochir (Wien) 150: 545500, 2008

13) Falleti MG, Maruff P, Collie A, Darby DG: Practice effects associated with the repeated assessment of cognitive function using the CogState battery at 10minute, one week and one month test-retest intervals. J Clin Exp Neuropsychol 28: 1095-1112, 2006

14) Folstein MF, Folstein SE, McHugh PR: “Mini-mental state". A practical method for grading the cognitive state of patients for the clinician. J Psychiatr Res 12: 189-198, 1975

15) Fukunaga A, Kawase T, Kashima H, Hashimoto J, Uchida K: Effects of habitual cigarette smoking on higher cortical function in patients with unruptured cerebral aneurysms. Neurol Med Chir (Tokyo) 42: 419-425, 2002

16) Fukunaga A, Uchida K, Hashimoto J, Kawase T: Neuropsychological evaluation and cerebral blood flow study of 30 patients with unruptured cerebral aneurysms before and after surgery. Surg Neurol 51: 132-138, 1999

17) Gottwald B, Wilde B, Mihajlovic Z, Mehdorn HM: Evidence for distinct cognitive deficits after focal cerebellar lesions. J Neurol Neurosurg Psychiatry 75: 1524-1531, 2004

18) Hoffmann M, Schmitt F: Cognitive impairment in isolated subtentorial stroke. Acta Neurol Scand 109: 14-24, 2004

19) Kashima H: [The application of neuropsychology to rehabilitation medicine]. Sogo Rehabilitation 13: 11-18, 1985 (Jpn, with Eng abstract)

20) Kashima H: [Neuropsychological assessment of dementia]. Ronen Seishin Igaku Zasshi 3: 253-260, 1992 (Jpn, with Eng abstract)

21) Kawase T, Bertalanffy H, Otani M, Shiobara R, Toya S: Surgical approaches for vertebro-basilar trunk aneurysms located in the midline. Acta Neurochir (Wien) 138: 402-410, 1996

22) Kawase T, Shiobara R, Toya S: Anterior transpetrosal-transtentorial approach for sphenopetroclival meningiomas: surgical method and results in 10 patients. Neurosurgery 28: 869-875, 1991

23) Kobayashi M, Takayama H, Suga S, Okazaki A, Mihara B: [Changes in proton magnetic resonance spectroscopy and Wechsler adult intelligence scale revised after clipping of unruptured aneurysms]. No Shinkei Geka 28: 691-698, 2000 (Jpn, with Eng abstract)

24) Lewis MC, Nevo I, Paniagua MA, Ben-Ari A, Pretto E, Eisdorfer S, Davidson E, Matot I, Eisdorfer C: Uncomplicated general anesthesia in the elderly results 
in cognitive decline: does cognitive decline predict morbidity and mortality? Med Hypotheses 68: 484492, 2007

25) Malm J, Kristensen B, Karlsson T, Carlberg B, Fagerlund $\mathrm{M}$, Olsson T: Cognitive impairment in young adults with infratentorial infarcts. Neurology 51: 433-440, 1998

26) Mollica CM, Maruff P, Vance A: Development of a statistical approach to classifying treatment response in individual children with ADHD. Hum Psychopharmacol 19: 445-456, 2004

27) Newman S, Stygall J, Hirani S, Shaefi S, Maze M: Postoperative cognitive dysfunction after noncardiac surgery: a systematic review. Anesthesiology 106: 572-590, 2007

28) Ogawa K, Toita T, Kakinohana Y, Tominaga D, Miyagi K, Yoshii Y, Horikawa A, Sawada S: A patient with improvement in short-term memory disturbance brought about by radiation therapy for germinoma involving Papez circuit. Radiat Med 17: 317-322, 1999

29) Ohue S, Oka Y, Kumon Y, Ohta S, Sakaki S, Hatakeyama T, Shiraishi T, Takeda S, Ohnishi T: Importance of neuropsychological evaluation after surgery in patients with unruptured cerebral aneurysms. Surg Neurol 59: 269-275, 2003

30) Papez JW: A proposed mechanism of emotion. 1937. J Neuropsychiatry Clin Neurosci 7: 103-112, 1995

31) Rasmussen LS: Postoperative cognitive dysfunction: incidence and prevention. Best Pract Res Clin Anaesthesiol 20: 315-330, 2006

32) Rasmussen LS, Larsen K, Houx P, Skovgaard LT, Hanning CD, Moller JT: The assessment of postoperative cognitive function. Acta Anaesthesiol Scand 45: 275-289, 2001

33) Ravizza SM, McCormick CA, Schlerf JE, Justus T, Ivry RB, Fiez JA: Cerebellar damage produces selective deficits in verbal working memory. Brain 129: 306-320, 2006

34) Riva D, Giorgi C: The cerebellum contributes to higher functions during development: evidence from a series of children surgically treated for posterior fossa tumours. Brain 123: 1051-1061, 2000

35) Silbert BS, Maruff P, Evered LA, Scott DA, Kalpokas M, Martin KJ, Lewis MS, Myles PS: Detection of cognitive decline after coronary surgery: a comparison of computerized and conventional tests. Br J Anaesth 92: 814-820, 2004

36) Snyder PJ, Werth J, Giordani B, Caveney AF, Feltner D, Maruff P: A method for determining the magnitude of change across different cognitive functions in clinical trials: the effects of acute administration of two different doses alprazolam. Hum Psychopharmacol 20: 263-273, 2005

37) Stoodley CJ, Schmahmann JD: Functional topography in the human cerebellum: a meta-analysis of neuroimaging studies. Neuroimage 44: 489-501, 2009

38) Temkin NR, Heaton RK, Grant I, Dikmen SS: Detecting significant change in neuropsychological test performance: a comparison of four models. J Int Neuropsychol Soc 5: 357-369, 1999

39) Yoshida K, Kawase T: Trigeminal neurinomas extending into multiple fossae: surgical methods and review of the literature. J Neurosurg 91: 202-211, 1999

40) Ziemus B, Baumann O, Luerding R, Schlosser R, Schuierer G, Bogdahn U, Greenlee MW: Impaired working-memory after cerebellar infarcts paralleled by changes in BOLD signal of a cortico-cerebellar circuit. Neuropsychologia 45: 2016-2024, 2007

Address reprint requests to: Shinya Ichimura, M.D., Department of Neurosurgery, Saiseikai Yokohamashi Tobu Hospital, 3-6-1 Shimosueyoshi, Tsurumi-ku, Yokohama, Kanagawa 230-8765, Japan. e-mail: shinya3917@gmail.com

\section{Commentary}

This study analyzed a series of patients with posterior cranial fossa lesions who underwent surgery either by a middle cranial fossa approach or by a lateral suboccipital approach. The authors found that middle cranial fossa approach group had a temporary cognitive impairment after the surgery, while lateral suboccipital approach group did not. They also found that the computerized test battery was more useful than the conventional test for detecting neuropsychological change.

Even in the patients who underwent surgery via the middle fossa approach, postoperative neuropsychological decline was improved within 3 months after the surgery. It seems that either approach to the posterior cranial fossa lesions is safe in terms of postoperative cognitive disorder, however, longerterm evaluation may be necessary by using standard neuropsychological tests such as Wechsler Intelligence Scale and Wechsler Memory Scale.

Takashi TокUтомі, M.D. Ichinomiya Neurosurgical Hospital Hita, Oita, Japan

The authors give us two useful information: 1. When we select surgical approaches for the posterior fossa lesions, we should consider that the cognitive function, especially short-term memory, may decline temporarily with the middle fossa approach and long operation time. 2. The present study also suggests that cerebellum may be related to cognitive function, based on the finding that long-lasting retraction of the cerebellum is an independent factor associated with the postoperative decline of short-term memory.

Yazhuo ZHANG, M.D.

Deputy Director

Beijing Neurosurgical Institute Beijing, P.R.C. 